\title{
Underlying Structural Aspects of DNA Repair
}

\author{
Abraham Minsky, Joseph Englander, and Daphna Frenkiel-Krispin
}

Departments of Organic Chemistry, The Weizmann Institute of Science, Rehovot 76100, Israel

The bacterium Deinococcus radiodurans survives exposure to more than 1.5 Mrad of ionizing irradiation or to extreme desiccation without lethality or mutagenesis. This tolerance derives from the ability of this organism to accurately mend numerous double-strand DNA breaks (DSBs), thus reassembling an intact genome from hundreds of fragments in a manner that restores chromosomal continuity. The only currently known mechanism that enables accurate repair of DSBs in bacteria is RecA-dependent homologous recombination, whereby information lost at a lesion is restored by a homologous DNA sequence that acts as a template. As such, DNA repair via homologous recombination strictly depends upon the ability of cellular systems to perform a rapid and efficient genome-wide search for homologous DNA sites. Yet, following extensive DNA fragmentation, no intact template remains. Homologous search conducted under such circumstances would necessarily entail repetitive re-inspection of randomly dispersed multiple DNA fragments, rendering the process inherently futile [1].

The high resistance of bacterial spores to irradiation and desiccation indicates that DSBs inflicted by these assaults in dormant spores are efficiently and accurately mended upon germination. Yet, DNA repair involving homologous search processes cannot occur in germinating spores, because bacterial spores regularly carry only one copy of their genome. Consequently, germinating spores lack the template required for homologous recombination-mediated accurate repair of DSBs. These and other considerations imply that the unique ability of both $D$. radiodurans and germinating spores to effectively repair double strand DNA breaks must depend upon novel strategies, which do not rely on RecA-dependent homologous recombination [2].

Structural studies conducted on D. radiodurans demonstrate that the chromatin in this organism adopts a ring-like toroidal morphology [3]. A similar toroidal organization of the chromatin is detected in mature as well as in germinating bacterial spores [4,5]. Within the tightly packed ringlike DNA morphology, ends of DNA fragments generated by DSBs are continuously held in close physical proximity, due to the restricted diffusion that is imposed by the tight packaging. We propose that the physical proximity of DNA ends enables accurate repair of DSBs in a templateindependent pathway, which is likely to proceed through nonhomologous DNA end-joining, as well as via homologous annealing of protruding DNA single strands, which might be present at the ends of DNA fragments $[3,4,6]$.

The notion that nonhomologous DNA end-joining within tightly-packed toroidal chromatin structures is operative in bacteria is substantiated by biochemical studies. DNA-binding proteins such as HU in D. radiodurans and SASP in bacterial spores were shown to specifically stabilize toroidal DNA structures both in-vitro and within bacterial cells. In addition, very small DNA ligases, which are capable of sliding along the DNA molecule in a tightly-packed context, were found to be induced in both $D$. radiodurans and spore-forming proteins. 
The observations presented here corroborate the notion that particular genome structures represent a crucial factor in the maintenance of DNA integrity in living systems exposed to harsh environmental conditions.
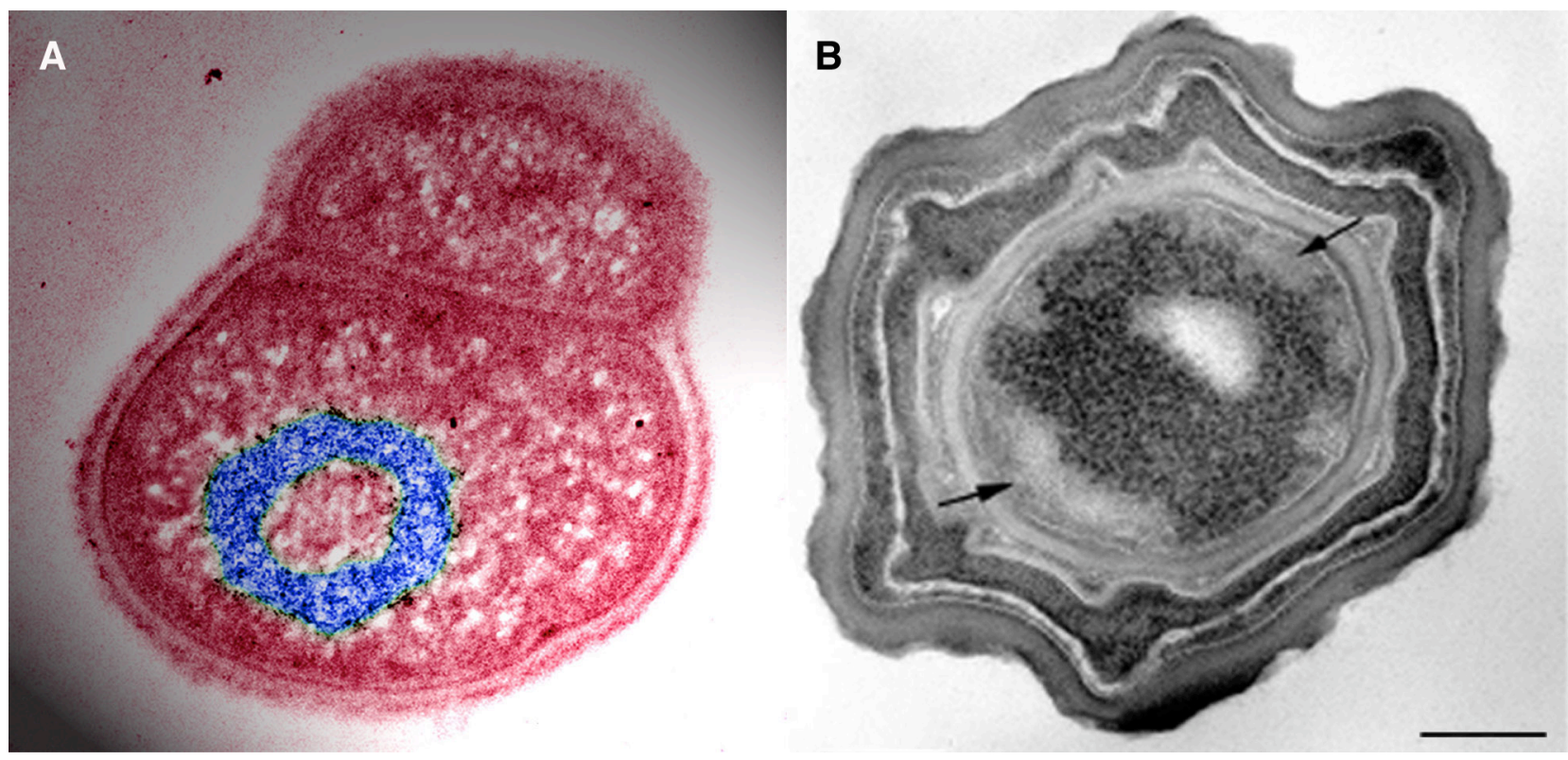

Figure 1. TEM of cryofixed D. radiodurans cell (A) and B. subtilis spore (B). (A): A colorenhanced micrograph of a $D$. radiodurans cell stained with the DNA-specific reagent osmiumammine- $\mathrm{SO}_{2}$. The toroidal structure of the chromatin is highlighted in blue. (B). Section of a mature $B$. subtilis spore, depicting the layers surrounding the sporal core. The densely stained particles in the center are ribosomes; ribosome-free spaces in the periphery of the spore core are indicated by arrows and contain chromatin. The ring-like organization of the sporal chromatin is indicated by specific DNA staining.

\section{References}

[1] M. J. Daly and K. W. Minton, Science 270 (1995) 1318.

[2] J. Englander et al., J. Bacteriol. 186 (2004) 5973.

[3] S. Levin-Zaidman et al., Science 299 (2003) 254.

[4] D. Frenkiel-Krispin et al., J. Bacteriol. 186 (2004) 3525.

[5] K. Ragkousi et al., J. Bacteriol. 182 (2000) 5556.

[6] A. Minsky, Mol. Microbiol. 50 (2003) 367.

[7] A. Minsky et al., Nat. Rev. Mol. Cell Biol. 3 (2002) 50. 